\section{Lit environments that promote health \\ and well-being
ait environments that promote health}

(1)

Karen van Creveld ${ }^{1}$ and Kevin Mansfield ${ }^{1}$

\begin{abstract}
The beneficial effects of light on human non-image forming processes are subject to much research interest and are outlined in this paper. Known mechanisms of impact include those on the circadian timing system, the arousal system and the affective system. An indication is made that it is important to consider the characteristics of the light field not just the light source when making recommendations concerning light of certain spectral quality. What types of light field are people exposed to on a regular basis and can these lit environments be considered beneficial? How much do these lit scenes differ in terms of spectral composition, as well as in light distribution patterns and illuminance levels, from those conditions that are currently suggested for optimising occupant health and wellbeing?

A representative set of natural and built lit environments were identified ranging from seascapes/landscapes to an urban square and various interior architectural environments. A photometric and colorimetric snapshot was measured during the midday hours, to replicate the visual field as viewed from the perspective of the observer. The measured spectral irradiance of light received at the cornea was converted into derived lighting metrics that are the subject of current recommendation: relative sensitivity curve $(V \lambda)$, vertical/horizontal illuminance ratio $\left(E_{v} / E_{h}\right)$, cylindrical illuminance $\left(E_{c}\right)$ and circadian stimulus $(C S)$. The data were analysed to demonstrate that the indications are that more natural lit environments are expected to be beneficial. Discussion follows about the relative importance of spectral composition, intensity and light distribution within the visual field and how these might combine to optimise our health and wellbeing.
\end{abstract}

\title{
Keywords
}

light, circadian, well-being

\section{Introduction}

In addition to enabling vision, light acts on various nonimage forming processes and hence impacts health and wellbeing at a core level. Known mechanisms of impact include those on the circadian system, the arousal system (arousal being the physiological and psychological state of being alert, awake and attentive) and the affective (emotional) system. Some areas of impact are better understood than others and have thus given rise to recommendations (from both academic and application practitioners) for types of lighting needed to maximise these impacts. The limitation of this approach is that there is the danger of creating unnatural visual environments that may inadvertently impact other fundamental mechanisms. The authors preferred approach is the application of full spectrum lighting viz. daylight as it satisfies known mechanisms without adversely impacting others.

Many claims are made about the beneficial effects of light of certain spectral composition. This has led in recent years to specific recommendations being put forward such as the impact of blue light on aspects of the circadian system. However, in addition to circadian functionality, various studies, including neuroimaging,(1) have shown that light activates many diverse areas of the brain, often simultaneously. Some of these areas are associated with known outcomes but as yet, neither the pathways linking these areas nor their true impact on downstream functioning is clear. Importantly, the spectral qualities of light most efficient for eliciting a response from most of these mechanisms, are currently unknown. Therefore, it seems counterproductive and possibly even harmful, to exclude certain wavelengths of light when considering optimal illumination for health and wellbeing.

\section{Circadian impacts of light}

Research into non-image forming effects of light gained momentum following the discovery in 2002 of the intrinsically photoreceptive Retinal Ganglion Cells (ipRGCs), an integral part of the retina of the eye.(2)(3)(4) These photoreceptors contain the photopigment melanopsin, which is most sensitive to light in the blue part of the spectrum, namely radiation of $480 \mathrm{~nm}$ wavelength.(5) Initially ipRGCs were thought to be a uniform type of ganglion cell, but more recent studies have identified five different types, possibly with different functional roles, designated M1 to M5.(6) Light incident on the retina is absorbed by the ipRGCs as well as by rods and cones and the ensuing electrical signals

\footnotetext{
${ }^{1}$ UCL Institute for Environmental Design and Engineering

\section{Corresponding author:}

Karen van Creveld, UCL Institute for Environmental Design and Engineering, The Bartlett school of Environment, Energy and Resources, Central House, 14 Upper Woburn Place, London, WC1H ONN

Email: karen.creveld17@ucl.ac.uk
} 
are transduced via the retinohypothalamic tract to various regions within the brain. The most noteworthy region for circadian impact is the suprachiasmatic nuclei (SCN) located within the hypothalamus.

The SCN are known as the body's biological master clock, generating and controlling endogenous ${ }^{1}$ circadian oscillations or circadian rhythms. Several output pathways from the SCN coordinate the activity of other clocks found in various sites throughout the body, influencing numerous processes, for example, hormone production, sleep timing and feeding patterns.(4) Human endogenous circadian rhythms function on a slightly longer timescale than 24 hours and regular exposure to periods of light and dark provide the primary environmental cues to entrain the circadian system to the solar day.(7)(8) From an evolutionary perspective, entrainment allows an organism to anticipate and prepare for the varying demands of the day/night cycle, optimising survival. On the other hand, a disrupted circadian system is associated with a range of adverse health outcomes.(9)(4)

One of the central biological functions controlled by the circadian system is the production of the hormone melatonin.(2) Light information signals reaching the SCN are conveyed to the pineal gland, where melatonin is synthesised from the neurotransmitter, serotonin. Melatonin is released into the bloodstream when ambient light levels fade in preparation for sleep and is understood to convey time of day messages throughout the body.

...the central role of melatonin is to synchronise the activation of many other physiological functions, not to the same time, but rather to the times in the 24-h cycle when they should occur.(10)

Melatonin plays an essential role in the regulation of the sleep-wake cycle and follows a daily rhythmic patternlevels rise with the onset of darkness and fall in anticipation of daytime. Melatonin is also associated with other vital processes including growth and puberty. Manipulation of melatonin levels has been shown in animal studies to affect the growth of different cancer types, especially hormone dependent breast cancer.(11)(12) Research shows that-

...melatonin reduces the incidence and growth of chemically induced mammary tumors, in vivo, and inhibits the proliferation and metastatic behavior of human breast cancer cells, in vitro.(13)

Both observations support the hypothesis that melatonin reduces the development of breast cancer mainly by interacting with œstrogen signalling pathways, whereby melatonin may either impact the production of circulating œstrogen hormones through the neuroendocrine system (hypothalamic - pituitary reproductive axis) and/or act on tumours at a cellular level.(13)

Studies have shown that exposure to light at night (LAN) suppresses the production of melatonin. Research into the threshold and spectral qualities of light needed to suppress melatonin at night have demonstrated that exposure to light in the blue portion of the spectrum, specifically at a wavelength of around 460nm, results in more effective suppression of melatonin, compared with polychromatic white light of the same intensity.(14) One of the agreed conclusions from this type of research is that-

... the human circadian system, as measured by acute melatonin suppression and phase shifting of dim light melatonin onset (DLMO), is a blue-sky detector with a peak spectral sensitivity close to $460 \mathrm{~nm}$.(14)

Recent studies have agreed that the retinal rod and cone photoreceptors and the ipRGCs are all involved in both visual and circadian phototransduction, the process by which light is converted into electrical signals.(15) However, as yet, the relative contributions of the various photoreceptors to innervate ${ }^{2}$ various areas of the human brain remains unknown.

\section{Effects of light on alertness}

In addition to the timing aspects of the circadian system, early studies showed that nightime exposure to white light over a wide range of illuminance levels impacted measures of alertness, as assessed by subjective ratings, quantification of slow eye movements (SEMS) and EEG analysis. All measures of alertness correlated highly with the degree of melatonin suppression and furthermore, the extent of the alerting response was determined by the intensity of the light stimulus.(16) Since the spectral sensitivity of the circadian system as measured by melatonin suppression has since been shown to peak in the blue region of the spectrum, more recent studies have investigated the impact of blue light on alertness at night.(14) One of the outcomes from this work is the claim that lower levels of short wavelength blue light will illicit the same alerting effects as significantly higher levels of polychromatic white light because of the underlying sensitivity of the ipRGCs to blue light.

However, studies by Vandewalle et al. using functional magnetic resonance imaging (fMR) looked at the impact of light on measures of alertness in rodents, conducted during the daytime, when melatonin levels are naturally low.(17) The results indicated that the light signals from the ipRGCs project to various areas of the hyperthalamus including the SCN, and from there to different parts of the brain associated with arousal (alertness). Whilst the pathways linking brain areas (that result eventually in cognition) are still unknown, the impact of light on daytime alertness appears to be influenced by brain mechanisms other than melatonin suppression.(14)

More recent studies have shown that exposure to red light of $630 \mathrm{~nm}$ can also increase objective and subjective measures of alertness, both at night and during the daytime.(18)(14) As the ipRGCs are insensitive to light in this region of the spectrum, it is suggested that the alerting effect of light may be due to the involvement of cone receptors sensitive to long wavelength light. This work supports the view that in addition to the ipRGCs, rod and cones are also involved in non-image forming functions and secondly, neural pathways other than those associated with melatonin suppression, are directly involved in measures of alertness. 


\section{Effects of light on the emotional system}

The impact of light on human emotions and wellbeing is the least well understood area of influence. To date most research has focused on using light to treat different forms of depression. Clinical evidence demonstrates that high levels of white light (2500 lux for 2 hours or 10000 lux for 30 minutes, administered in the morning and measured at eye level is the preferred treatment for seasonal affective disorder (SAD). (19) Bright white light therapy has also been shown to be effective in treating other forms of depression, including bipolar depression and chronic depression but unlike SAD treatment, as yet, no specific guidelines for light levels or duration have been agreed. (20) Currently, definitive causes of SAD as well as the mechanism of action of bright light treatment, remain unclear. However, both low levels of circulating serotonin and disrupted sleep-wake disorders are features of the condition, suggesting the involvement of both the serotonin and circadian regulatory systems.(21)

These two systems are extensively intertwined; they share anatomical connections and their signalling networks regulating gene expression, overlap. Both systems are understood to contribute to similar types of mood and developmental disorders such as seasonal affective disorder (SAD), bipolar disorder, major depression and autism spectrum disorder.(21) Additionally, both systems are affected by exposure to bright light.(22)

The neurotransmitter serotonin originates in the brainstem and projects to other brain regions (including limbic structures), known to regulate a wide range of physical and emotional responses to environmental stimulation, impacting mood, appetite, sleep and overall sense of wellness. In addition, serotonin is the precursor of the hormone melatonin and also exhibits rhythmicity, exhibiting both daily and seasonal variations, with lowest levels recorded during the winter months. This appears to be directly related to the prevailing availability of bright sunlight. (20)

\section{Full spectrum lighting}

Whilst targeted blue light exposure has been shown to be effective in some studies conducted under laboratory conditions,(14) the problem that emerges is that this constitutes only a very small portion of the electromagnetic spectrum. Over millions of years of evolution, humans have adapted and thrived under a regular rhythm of bright daylight and true darkness. And although daylight conditions vary with time of day, season and latitude, all daylight conditions contain varying proportions of all the wavelengths of the visible spectrum plus others. Exclusion of some of the constituent wavelengths of full spectrum light or indeed, preferential doctoring of the light environment to influence certain known biological responses may result in inadvertent and unwelcome effects elsewhere. The true impact and benefit of exposure to full spectrum light on human biology is not yet well understood and is the subject of ongoing and vital research. However, disruption of both the timing and the characteristics of environmental light is understood to impact negatively on various biological functions from poor sleep quality to increased risks for more serious diseases.(9)

Most of the claims above refer solely to the characteristics of the light source without considering the overall light field to which people are exposed. The lit environments through which people move, work and live are a combination of source (both natural and artificial) and light reflected from architectural surfaces and volumes. If the photometric and colorimetric characteristics of this exposure are understood then they can be compared to design recommendations that are being suggested by lighting researchers and/or application guidelines such as the WELL Building Standard.(23)

\section{Proposed metrics}

Whilst extensive research has been undertaken over the past three decades to identify and quantify the impact of light on various non-image forming functions, to date "... it is not yet possible to predict the non-image forming impact of a given illuminant based on its intensity and spectral composition. However, some guidance is possible."(7). The range and complexity of non-image forming functioning means that it is unlikely that a single unit of measurement will be able to explain all the effects of light under all conditions. Having said that, significant efforts have been made to define units of measurement to quantify certain aspects of non-image forming functioning, especially with regard to light's effect on the circadian system. In general these proposals are based on two separate approaches. One approach estimates the ability of light to influence the production of melatonin by the pineal gland as circulating melatonin is considered by many researchers to be a marker of circadian timing. The second proceedure is to apply the different spectral sensitivity functions (of the individual photoreceptor types) to the the irradiance (Watts per square metre $\left(W / m^{2}\right)$ received at the eye, in order to estimate the extent to which a given light source will activate each of the different types of photoreceptors in the human retina. A point worth making is that whilst all the photoreceptors are understood to be involved in both visual and nonimage forming functionality, their relative contributions remain unknown. These new metrics have emerged because traditional photometry, based as it is on the functioning of the human rods and cones, does not include the behaviour of the ipRGCs which are most sensitive to light within the blue portion of the electromagnetic spectrum. Regardless of the metric used, researchers now agree that all known photoreceptors in the retina, the three cone types, the rods and the ipRGCs are all involved in both visual and nonimage forming functions.(7)(24)(15) In this regard, Rea (25) has proposed a metric termed the universal luminous efficiency function $(U \lambda)$ which combines the full range of spectral efficiencies of the five known photoreceptors in the human retina. He proposes that this metric provides a more accurate representation of the human retina's response to electromagnetic radiation than $(V \lambda)$.

Circadian stimulus The Circadian Stimulus metric $(C S)$ is a mathematical model proposed by the Lighting Research Centre at Rensselaer Polytechnic Institute to explain the impact of light on the circadian system. This model is based on extensive existing literature on the anatomy and physiology of retina/brain structures plus experimental investigations into the suppression of melatonin at night by exposure to different types of light stimulus.(26) 
Using this model, the density of light measured at the cornea is converted into circadian light (CLA), which is comparable to conventional photopic illuminance (i.e. the amount of luminous flux incident upon a given surface) but weighted by the spectral sensitivity of the human circadian system as measured by acute melatonin suppression after a 1 -h exposure. The CLA value can then be used to determine circadian stimulus (CS), which reflects the effectiveness of the spectrally weighted density of light incident at the eye from no suppression, or threshold $(\mathrm{CS}=0.1)$, to saturation $(\mathrm{CS}=0.7)$.(15)

The $C S$ metric is an attempt to quantify how much light of a specific spectral composition will be needed to suppress melatonin after exposure for 1 hour. The WELL Standard has recently (Feb 2019) included the option to use the Circadian Stimulus $(C S)$ metric as an alternative method for determining compliance with Circadian Lighting Design, one of the fundamental features of the Standard. The WELL Standard is a performance-based system of measuring, certifying and monitoring the built environment that focuses primarily on optimising human health and wellbeing.(23)

\section{Method}

We ask the question, therefore, what are the photometric and colorimetric characteristics of the light field to which people are routinely exposed? Can Circadian Stimulus $(C S)$ characterise such lighting settings?

\section{Lighting settings}

Nine locations in and around London were chosen to be representative of urban and architectural lit environments. The criteria for selection was that they should be environments where people-office workers, retail staff, outdoor workers-would routinely spend long periods of time and that they should range from open landscape and urban settings to enclosed architectural settings. The intent was to choose settings which ranged from highly 'natural' to those more 'artificial' or 'synthetic'. The following list of settings is arranged approximately in order from the most natural to the most artificial/synthetic and describes the setting, its location and typical users of the setting-

1. Landscape East Sussex-agricultural workers.

2. Square Fitzroy Square, London-street cleaners.

3. Toplit Study space, SOAS, London—students.

4. Domestic Living room, London-homeowners.

5. Retail Bookshop, London—sales staff.

6. Office 1 Two person office, London-office workers.

7. Office 2a Open-plan office, London-office workers.

8. Office 2b Open-plan office, London-office workers.
9. Healthcare Hospital waiting, London-reception staff.

Many of the settings had a significant daylight component and the intention being only to secure a snapshot of the light field, measurements were taken under overcast (8/8 okta) and sunny (2-3/8 oktas) sky conditions. ${ }^{3}$ Measurements were taken between approximately $11.00 \mathrm{a} . \mathrm{m}$. and 1.00 p.m. during June 2019.

The overcast settings are shown in Figure 1 and the sunny in Figure 2.

\section{Photometric measurements}

Measurements were made at each location at $1.5 \mathrm{~m}$ above floor level in the principal direction of view of a long-term observer.

Spectral measurements A spectral measurement was made using a portable spectrophotometer (AsenseTek Lighting Passport Pro) linked via Bluetooth to associated software (Spectrum Genius Essence Plus) installed on an iPhone 6. Connection of the device to a web portal (www.spectrumgenius.com) allowed the download of light spectrum, a variety of colour metrics and Circadian Stimulus (CS) at each location.

Cubic illuminances Cubic illuminances(27) at the observer position (the planar illuminance on each of six sides of an infinitesimally small cube) were measured using a Konica Minolta T-10A illuminance meter. The illuminance $E_{x}$ corresponded with the principal direction of view (Figure 3). This allowed a variety of illumination metrics to be calculated.

A reference photograph was taken and any extraneous factors that may have impacted on the measurements were noted.

\section{Results}

In order to explore the results we separated the data set into two subsets- the overcast setting and the sunny setting (Tables 1 and 2).

The portable spectrophotometer, through the associated web portal, is able to generate the light spectrum, a variety of colour metrics (including CIE $1931 x$ and $y$ chromaticity coordinates and Correlated Colour Temperature) and the Circadian Stimulus $(C S)$. For our purposes we choose only to quote $C S$ in Tables 1 and 2 for each setting. We also show the six cubic illuminances (in $l x$ ) measured at each setting $\left(E_{x}, E_{-x}, E_{y}, E_{-y}, E_{z}\right.$ and $\left.E_{-z}\right)$.

Note that the healthcare setting was repeated in both data sets as, being in a basement, daylight was excluded. The data was imported into the $\mathrm{R}$ statistical software environment and bar plots for Circadian Stimulus $(C S)$, the ratio of vertical to horizontal illuminance $\left(E_{v} / E_{h}\right)$, cylindrical illuminance $\left(E_{c}\right)$ and illuminance at the eye $\left(E_{\text {eye }}\right)$ were generated for the two subsets. 


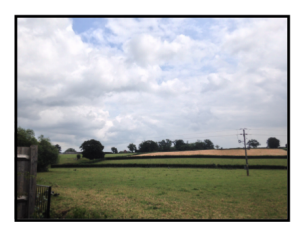

Landscape

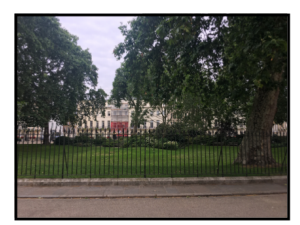

Square

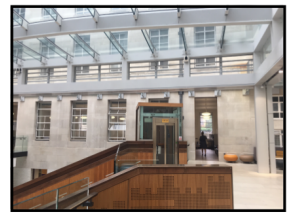

Toplit

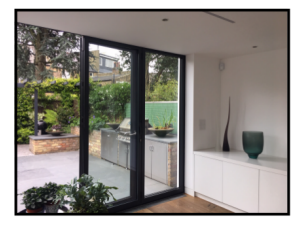

Domestic

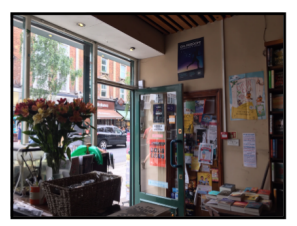

Retail

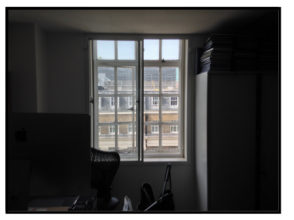

Office 1

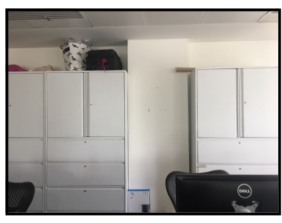

Office $2 \mathrm{a}$

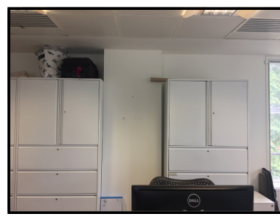

Office $2 b$

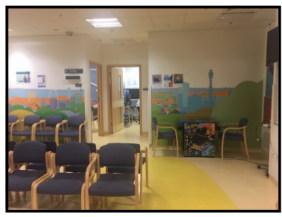

Healthcare

Figure 1. Settings (overcast sky condition)

\section{Analysis}

\section{Circadian Stimulus (CS)}

A bar plot for each setting (overcast and sunny) is shown in Figures 4 and 5.

Prepared using sagej.cls
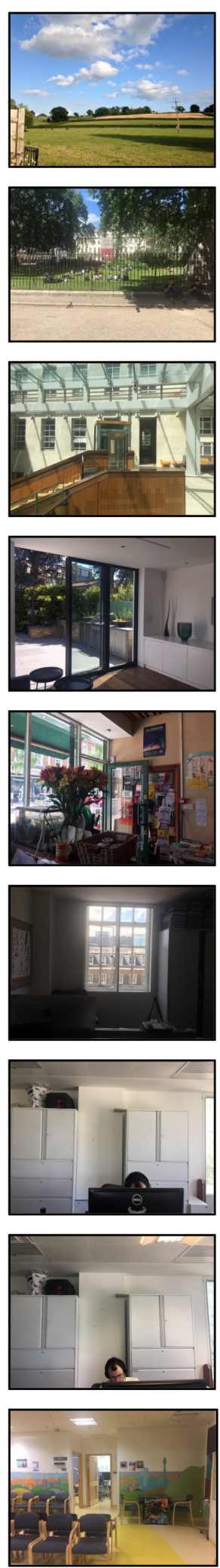

Office 1

Office $2 b$

Square

Toplit

Domestic

Retail

Office $2 \mathrm{a}$

Healthcare
Landscape

Figure 2. Settings (sunny sky condition)

Figueiro et al. suggest that a $C S$ of 0.3 or greater, received during the early part of the day and measured at 


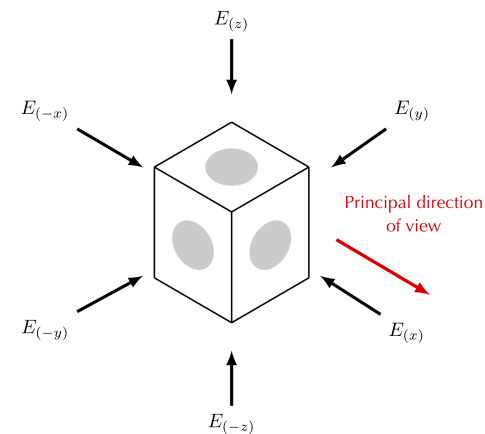

Figure 3. Cubic illuminance showing orientation

Table 1. The overcast setting showing Circadian Stimulus and cubic illuminances $(l x)$.

\begin{tabular}{llllllll}
\hline SETTING & $C S$ & $E_{x}$ & $E_{-x}$ & $E_{y}$ & $E_{-y}$ & $E_{z}$ & $E_{-z}$ \\
\hline Landscape & 0.69 & NA & NA & NA & NA & NA & NA \\
Square & 0.68 & 7980 & 8580 & 13870 & 8290 & 28960 & 3280 \\
Toplit & 0.55 & 1604 & 1181 & 1668 & 1125 & 3830 & 885 \\
Domestic & 0.53 & 1464 & 2157 & 4640 & 690 & 5410 & 624 \\
Retail & 0.43 & 558 & 74 & 312 & 144 & 380 & 86 \\
Office 1 & 0.37 & 268 & 68 & 104 & 82 & 102 & 57 \\
Office 2a* & 0.1 & 123 & 224 & 101 & 363 & 147 & 55 \\
Office 2b & 0.11 & 190 & 228 & 123 & 719 & 623 & 115 \\
Healthcare & 0.12 & 194 & 205 & 351 & 218 & 352 & 144 \\
\hline * Lights OFF & & & & & & &
\end{tabular}

Table 2. The sunny setting showing Circadian Stimulus and cubic illuminances $(l x)$.

\begin{tabular}{|c|c|c|c|c|c|c|c|}
\hline SETTING & $C S$ & $E_{x}$ & $E_{-x}$ & $E_{y}$ & $E_{-y}$ & $E_{z}$ & $E_{-z}$ \\
\hline Landscape & 0.7 & 51100 & 28000 & 13810 & 48900 & 119900 & 10240 \\
\hline Square & 0.68 & 10130 & 73200 & 45600 & 12460 & 110000 & 14300 \\
\hline Toplit & 0.68 & 8850 & 18260 & 15570 & 12000 & 52300 & 10340 \\
\hline Domestic & 0.59 & 1084 & 1040 & 2241 & 630 & 1804 & 423 \\
\hline Retail & 0.34 & 202 & 79 & 377 & 137 & 313 & 40 \\
\hline Office 1 & 0.4 & 348 & 83 & 115 & 114 & 113 & 54 \\
\hline Office $2 a^{*}$ & 0.11 & 135 & 189 & 113 & 429 & 161 & 56 \\
\hline Office $2 b^{\dagger}$ & 0.06 & 198 & 227 & 135 & 792 & 712 & 129 \\
\hline Healthcare & 0.12 & 194 & 205 & 351 & 218 & 352 & 144 \\
\hline
\end{tabular}

* Lights OFF

${ }^{\dagger}$ Lights ON

the eye position, is effective for stimulating the circadian system.(15). Figures 4 and 5 show very clearly that the deep open plan office setting (Office $2 a$ and $2 b$ ) in both the overcast and sunny conditions and the basement hospital reception (Healthcare), receive far less than 0.3 Circadian Stimulus and therefore would be considered to fall short of providing lighting to support healthy circadian functioning. It is interesting to note that the bookshop (Retail) generates a low $C S$ value on a sunny day-this is because the staff have chosen to install an awning to provide exterior sun shading. (The reason given for this was to limit heat gain rather than reduce daylight ingress). On the other hand, in both the overcast and sunny settings there is a substantial daylight component in the study space (Top-lit) arrangement, providing close to the maximum Circadian Stimulus of 0.7 . Settings with large windows providing generous side lit conditions, such as the single occupancy office (Office 1) and the bookshop, provide at least $C S=0.3$. Generally as we move from more natural settings to more artificial/synthetic settings (i.e. from left to right) the $C S$ potential decreases. Whilst this metric alone cannot fully describe the circadian benefits provided by a lit environment, it is nonetheless a useful tool to estimate the impact of light in different settings on one aspect of our non-image forming functioning.

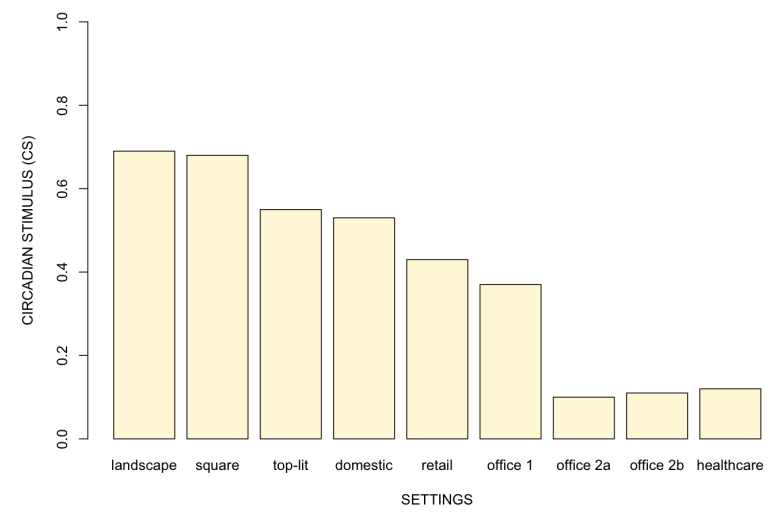

Figure 4. Circadian Stimulus for each setting (overcast)

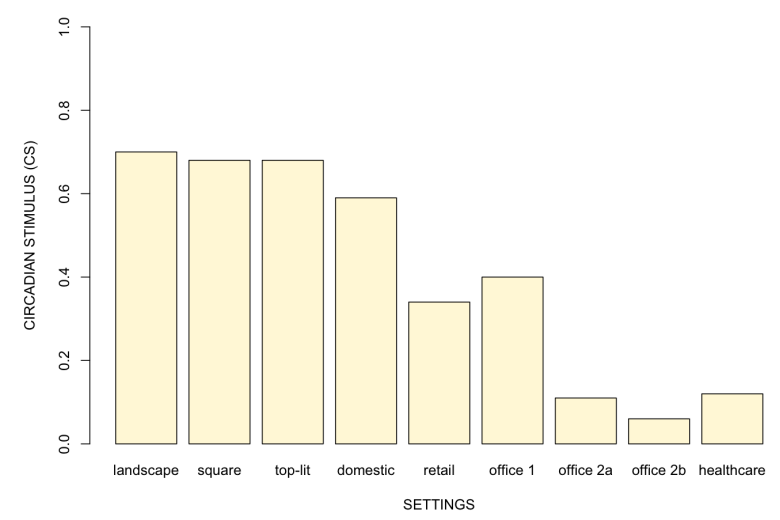

Figure 5. Circadian Stimulus for each setting (sunny)

\section{Vertical/horizontal illuminance ratio $\left(E_{v} / E_{h}\right)$}

Figueiro et al.(28) further claim that, when designing with $C S$, account must also be taken of the distribution of light and recommend that luminaire installations be so designed to provide the best ratio of vertical illuminance, $E_{v}$ to horizontal illuminance $E_{h}$. For each setting the ratio of $E_{v}$ to $E_{h}$ was calculated (equivalent to $E_{x} / E_{z}$ ). A bar plot for each setting (overcast and sunny) is shown in Figures 6 and 7. 


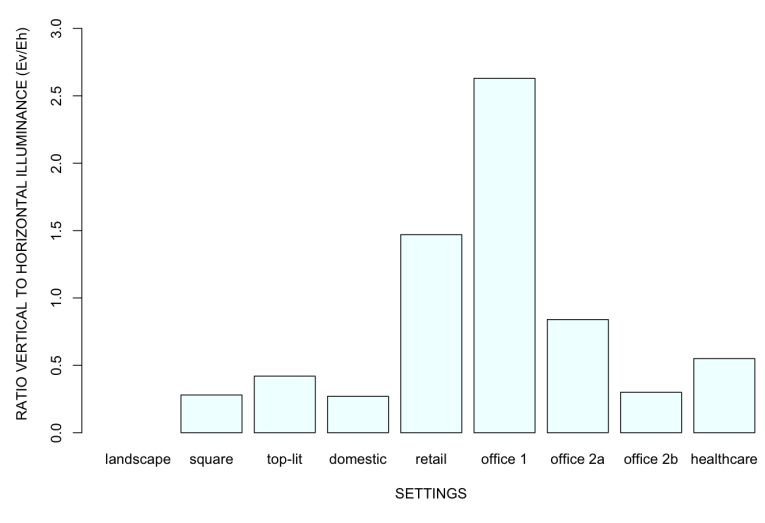

Figure 6. Vertical/horizontal illuminance ratio for each setting (overcast)

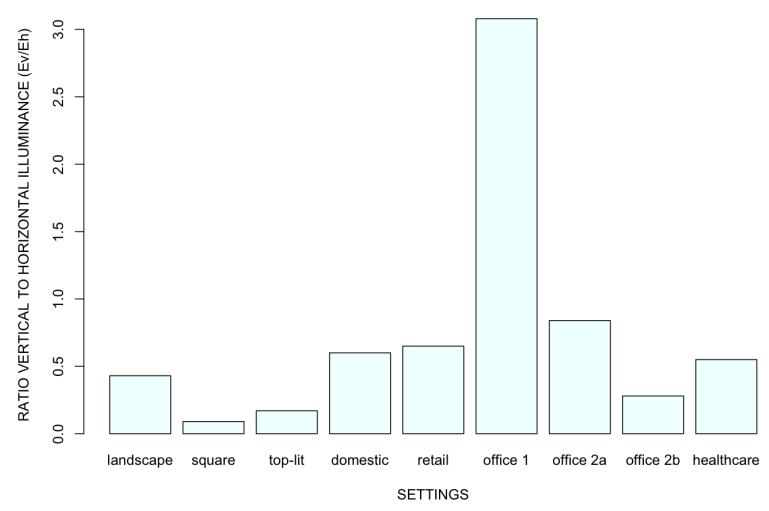

Figure 7. Vertical/horizontal illuminance ratio for each setting (sunny)

The results demonstrate that the highest ratio of vertical to horizontal illuminance is seen in the single occupancy office (Office 1) during both the sunny and overcast conditions. This may be due to the high levels of inter-reflected light, entering the space from the relatively large side window. Also this office is a comparatively small space with light coloured walls, improving inter-reflection and providing an overall impression of lightness. The $E_{V} / E_{H}$ is also relatively high in the bookshop (Retail) during the overcast condition only, probably for similar reasons. The $E_{V} / E_{H}$ ratio in the deep open plan office (Office $2 \mathrm{a}$ and $2 \mathrm{~b}$ ) is fairly low during both sunny and overcast conditions especially when the overhead downlights are switched on, suggesting a less comfortable visual environment. All top lit spaces including the domestic space (which has a large glazed rooflight), result in very low $E_{V} / E_{H}$ ratios. These results point to a discrepancy between the observed light distributions (see Figures 1 and 2) and the metric used here to describe them. From observation, none of the top lit spaces appear to suffer from overtly disproportionate overhead lighting yet the results seem to suggest that this would be the case. This raises the possibility that this metric may be suitable only for smaller interior spaces where any daylight contribution enters only from side-lit windows and is not appropriate for large top-lit daylight environments. Tregenza(29) points to experimental evidence that "... supports the existence of 'normal' brightness distributions" in the most common interior space-the rectilinear room. When people are asked to adjust the lighting to their preference or select from a preset luminance pattern, they choose distributions characteristic of daylit spaces.

\section{Cylindrical illuminance $\left(E_{c}\right)$}

People prefer 'light and airy' spaces and it is felt that mean cylindrical illuminance correlates best with the overall impression of brightness in a space(30) and has been proposed as an index of the perceived adequacy of ambient illumination.(27). For each setting the mean cylindrical illuminance $\left(E_{c}\right)$ was calculated (equivalent to $\left(E_{x}+\right.$ $\left.E_{-x}+E_{y}+E_{-y}\right) / 4$ ). A bar plot of mean cylindircal illuminance for each setting (overcast and sunny) is shown in Figures 8 and 9.

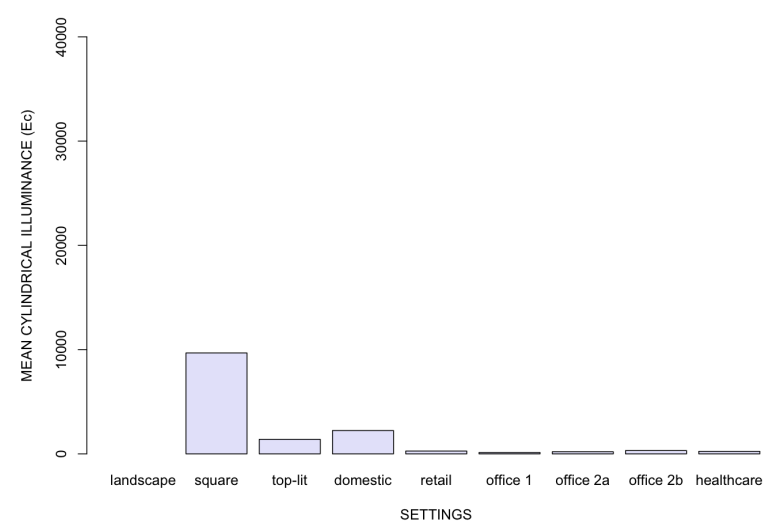

Figure 8. Cylindrical illuminance ratio for each setting (overcast)

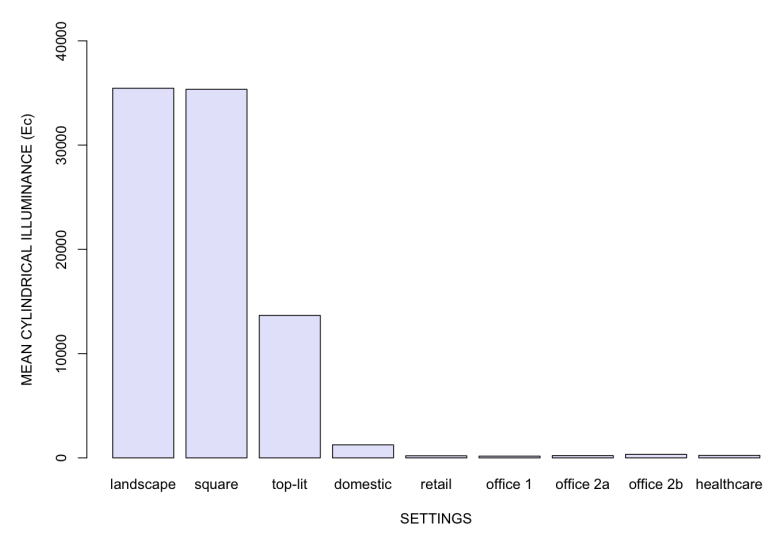

Figure 9. Cylindrical illuminance ratio for each setting (sunny)

The results show that the exterior settings achieve substantially higher cylindrical illuminance ratings than any 
of the interior environments, during both sunny and overcast conditions. Of all the interior settings, the top lit spaces perform best, although at a markedley lower level than the exterior settings, where the full benefit of the extensive sky dome is evident. It is also obvious that sun position dramatically influences the cylindrical illuminance results and the presence of sunlight is clearly seen in the tabulated individual illuminances-see Table 2. The domestic setting performs better than the top lit space during the overcast conditions with this metric and this may be explained by the presence of the fully glazed wall together with the large glazed roof. The combination of high levels of daylight and light coloured wall finishes provides good inter-reflection and results in balanced and uniform threedimensional illuminance.

\section{Illuminance at the eye ( $\left.E_{\text {eye }}\right)$}

Figueiro et al.(28) also urge designers to design for vertical (= corneal) illuminance at the eye not just the illuminance on the horizontal workplane. This is the the illuminance at a point normal to the direction of view and may be used as an indicator of the state of visual adaptation.(27) It is equivalent to $E_{x}$. A bar plot of illuminance at the eye for each setting (overcast and sunny) is shown in Figures 10 and 11. Note that the $y$-axis is plotted on a logarithmic scale.

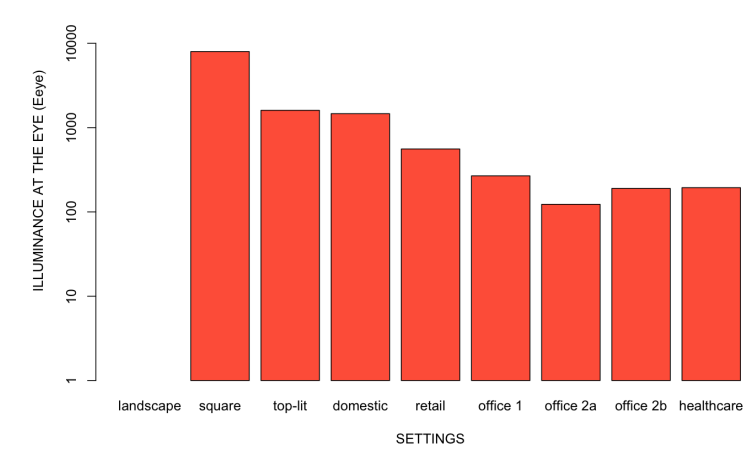

Figure 10. Illuminance at the eye for each setting (overcast)

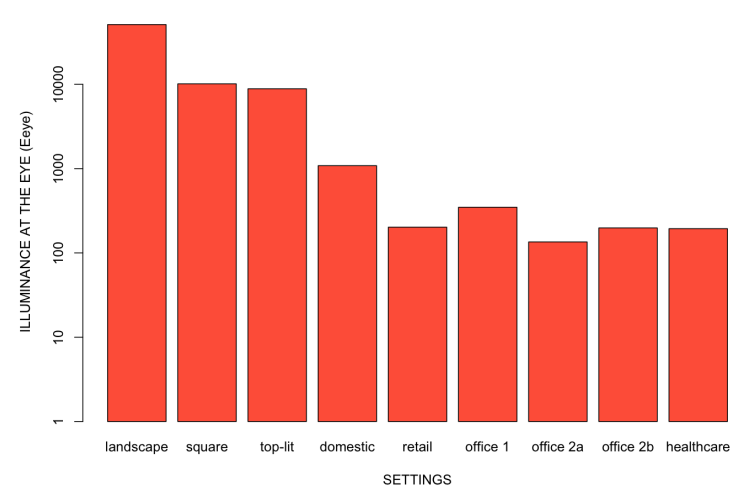

Figure 11. Illuminance at the eye for each setting (sunny)

The striking feature of these results is that the natural and top-lit settings produce high illuminance at the eye leading to a light-adapted visual system which reduces to a plateau as you move to more architectural settings (Office 1, 2a, 2b and Healthcare). As Tregenza suggests,(29) the living room, the classroom, the office are "... the kind of rooms in which most people spend many of their daytime hours and where many of the significant events of life take place.” Why therefore do we design environments where people have such low corneal illuminance?

\section{Discussion and Conclusion}

Four metrics have been used to describe the light fields afforded by various architectural settings with the aim of determining which conditions offer occupants the most benefit for health and wellbeing. Circadian Stimulus $(C S)$ and mean cylindrical illuminance $\left(E_{c}\right)$ seem to be running roughly in tandem and show generally that those environments with high levels of daylight score highly in both metrics and hence can be considered to support certain known measures of non-image forming and visual functioning. These findings are in contrast with those seen in the ratio of vertical to horizontal illuminance $\left(E_{v} / E_{h}\right)$, where higher values are the consequence of very specific architectural geometries. This metric may not be so useful in characterising lit environments that promote health and well-being. The illuminance at the eye $\left(E_{\text {eye }}\right)$ powerfully describes the difference between natural and open urban environments compared to architectural interiors.

This study has investigated a finite number of natural and built lit environments where people routinely spend long periods of time and as such embodies certain limitations. The paucity of settings means that the true diversity of lit environments found in contemporary life could not be accurately represented. Additionally, this study captured only a momentary snapshot of the visual field during two daylight conditions and hence was unable to describe the full variation of the visual field over time. Possible technical drawbacks of the measuring equipment used is also acknowledged. The spectrophotometer and associated software excluded information about how the metrics were calculated and whilst the device appeared to be cosine corrected, confirmation was not available. This is an example of the potential problems associated with readily available technology and points to the need for such equipment to undergo careful verification to ensure confidence in the results. Future work in this field would benefit from longer term studies incorporating a wider range of environments including those where the lighting has been specifically designed to address circadian needs.

Wilkins(31) states that "...the human visual system evolved to process images from nature" and we would claim that the results reported here point to the primacy of the natural daylit environment. Wilkins outlines that discomfort is caused by scenes in which the "... spatial, chromatic or temporal features depart from those usually found in nature."(31) The Circadian Stimulus ( $C S$ ) metric can only really describe the temporal variation in brightness which is largely circadian - it does not say anything about the spatial structure and colour contrast in the light field and these are probably better described by illumination metrics related to preference or appearance. Circadian lighting design using 
$C S$ is best restricted to windowless environments such as industrial control rooms or nursing bases where staff spend long periods of time and work unsocial hours.

As Boyce points out,(10) there exists a fundamental contradiction regarding the use of light metrics which can be precisely undertaken and be seen to be very accurate measures of physical properties. However, when attempting to measure how humans respond to light, any outcomes are, at best, an approximation of effect. Because of the complexity and adaptability of the human visual system and the large differences between individuals, precise measurement of light's impact on vision is unlikely. This uncertainty is substantially heightened when attempting to quantify the effect of light on non-image forming functions. Whilst significant advances have been made in deciphering the mechanisms of human biology and the role of light in maintaining health and wellbeing, research in this field is very much in its infancy.

\section{Declaration of conflicting interests}

The author(s) declared no potential conflicts of interest with respect to the research, authorship, and/or publication of this article.

\section{Funding}

The author(s) received no financial support for the research, authorship, and/or publication of this article.

\section{Notes}

1. endogenous-Having an internal cause or origin.

2. innervate-To supply an organ with nerves.

3. okta-Cloud cover expressed in eighths.

\section{List of Figures}

Figure 1. Settings (overcast sky condition)

Fig-1.tiff

Figure 2. Settings (sunny sky condition)

Fig-2.tiff

Figure 3. Cubic illuminance showing orientation

Fig-3.tiff

Figure 4. Circadian stimulus for each setting (overcast)

Fig-4.tiff

Figure 5. Circadian stimulus for each setting (sunny)

Fig-5.tiff

Figure 6. Vertical/horizontal illuminance ratio for each setting (overcast)

Fig-6.tiff

Figure 7. Vertical/horizontal illuminance ratio for each setting (sunny)

Fig-7.tiff

Figure 8. Cylindrical illuminance ratio for each setting (overcast)

Fig-8.tiff

Figure 9. Cylindrical illuminance ratio for each setting (sunny)

Fig-9.tiff

Figure 10. Illuminance at the eye for each setting (overcast) Fig-10.tiff

Figure 11. Illuminance at the eye for each setting (sunny)

Fig-11.tiff

\section{References}

[1] Vandewalle G, Maquet P and Dijk DJ. Light as a modulator of cognitive brain function. In Trends Cogn Sci 2009; 13(10):429438.

[2] Thapan K, Arendt J and Skene DJ. An action spectrum for melatonin suppression: Evidence for a novel non-rod, noncone photoreceptor system in humans. In J Physiol 2001; 535(1):261-267.

[3] Berson DM, Dunn FA and Takao M. Phototransduction by retinal ganglion cells that set the circadian clock. In Science 2002; 295(5557):1070-1073.

[4] Foster R and Kreitzman L. Circadian rhythms: A very short introduction. Oxford: Oxford University Press, 2017, p.28, 50 and 56.

[5] Hattar S, Liao HW, Takao M DMB et al. Melanopsincontaining retinal ganglion cells: Architecture, projections, and intrinsic photosensitivity. In Science 2002; 295(5557):10651070.

[6] Schmidt TM, Shih-Kuo Chen and Hattar S. Intrinsically photosensitive retinal ganglion cells: Many subtypes, diverse functions. In Trends Neurosci 2011; 34(11):572-580.

[7] Lucas RJ, Peirson SN, Berson DM et al. Measuring and using light in the melanopsin age. In Trends Neurosci 2014; 37(1):19.

[8] Wright KP, McHill AW, Birks BR et al. Entrainment of the human circadian clock to the natural light-dark cycle. In Curr Biol 2013; 23(16):1554-1558.

[9] Brainard J, Gobel M, Scott B et al. Health implications of disrupted circadian rhythms and the potential for daylight as therapy. In Anesthesiology 2015; 122(5):1170-1175.

[10] Boyce P. Human factors in lighting. Boca Raton:CRC Press, 2014, p.95, 105 and 243.

[11] Stevens R and Rea M. Light in the built environment: potential role of circadian disruption in endocrine disruption and breast cancer. In Cancer Causes and Control 2001; 12: 279-287.

[12] Schernhammer E and Schulmeister K. Light at Night and Cancer Risk. In Photochemistty and Photobiology 2004; 79(4): 316-318

[13] Cos S, Gonzalez A, Martnez-Campa C, et al Estrogensignaling pathway: A link between breast cancer and melatonin oncostatic actions. In Cancer Detection and Prevention 2006; 30: $118-128$.

[14] Figueiro MG, Nagare R and Price LLA. Non-visual effects of light: How to use light to promote circadian entrainment and elicit alertness. In Light Res Technol 2018; 50(1):38-62. 
[15] Figueiro MG. Disruption of circadian rhythms by light during day and night. In Curr Sleep Medicine Rep 2017; 3:76-84.

[16] Cajochen C, Zeitzer J, Czeisler C et al Doseresponse relationship for light intensity and ocular and electroencephalographic correlates of human alertness. In Behavioural Brain Research 2000; 115(1): 75-83

[17] Vandewalle G,Balteau E, Phillips C et al Daytime Light Exposure Dynamically Enhances Brain Responses. In Current Biology 2006; 16: 1616-1621

[18] Figueiro M, Bierman A, Plitnick B et al Preliminary evidence that both blue and red light can induce alertness at night. In BMC Neuroscience 2009; 10:105

[19] Raynham P. The SLL Code for Lighting The Society of Light and Lighting, CIBSE, 2012, p 17, 39

[20] Pail G, Huf W, Pjrek E, et al Bright-light therapy in the treatment of mood disorders. In Neuropsychobiology. 2011; 64(3):152-162

[21] Ciarleglio CM, Resuehr HES and McMahon DG. Interactions of the serotonin and circadian systems: Nature and nurture in rhythms and blues. in Neuroscience. 2011; 197:8-16

[22] Young S N, How to increase serotonin in the human brain without drugs. In J Psychiatry Neurosci. 2007; 32(6):394-399

[23] WELL building standard. International WELL Building Institute (IWBI), 2017.

[24] The SLL Lighting Handbook In The Society of Light and Lighting, CIBSE, 2018 , p. 448

[25] Rea M. The lumen seen in a new light: Making distinctions between light, lighting and neuroscience. In Lighting Res. Technol. 2015; 47: 259-280

[26] Rea MS, Figueiro MG, Bullough JD et al A model of phototransduction by the human circadian system. In Brain Res Rev, 2005; 50:213-228.

[27] Cuttle C. Cubic illumination. In Ltg Res Tech, 1997; 29(1):114.

[28] Figueiro MG, Gonzales K and Pedler D. Designing with Circadian Stimulus. In LDA October 2016

[29] Tregenza P and Wilson M. Daylighting: Architecture and lighting design. Oxford:Routledge, 2011, p.16.

[30] Tregenza $\mathrm{P}$ and Loe D. The design of lighting. Oxford:Routledge, 2013, p.21.

[31] Wilkins AJ. A physiological basis for visual discomfort: Application in lighting design. In Ltg Res Tech, 2016; 48:4454. 